\title{
Outcomes of hospitalized patients with COVID-19 according to level of frailty
}

Eva María Andrés-Esteban ${ }^{1,2}$, Manuel Quintana-Diaz ${ }^{1,3}$, Karen Lizzette Ramírez-Cervantes ${ }^{1,4}$, Irene Benayas-Peña ${ }^{1}$

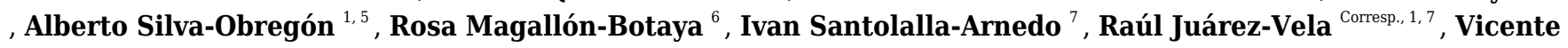
Gea-Caballero $^{8,9}$

${ }^{1}$ Grupo PBM, Instituto de Investigación-IdiPaz, Madrid, Madrid, Spain

2 Universidad Rey Juan Carlos, Madrid, Madrid, Spain

3 Servicio de Medicina Intensiva, Hospital Universitario La Paz, Madrid, Madrid, Spain

4 Departamento de Prevención, Asociación Española contra el Cáncer, Madrid, Madrid, Spain

5 Servicio de Medicina Intensiva, Hospital Universitario de Guadalajara, Guadalajara, Guadalajara, Spain

6 Departamento de Medicina, Psiquiatría y Dermatología, Universidad de Zaragoza, Zargoza, Aragón, Spain

7 Universidad de La Rioja, Centro de Investigación Biomédica de La Rioja-CIBIR, Logroño, La Rioja, Spain

8 Nursing School La Fe., Adscript center of Universidad de Valencia., Valencia, Valencia, Spain

9 Research Group GREIACC, Health Research Institute La Fe., Valencia, Valencia, Spain

Corresponding Author: Raúl Juárez-Vela

Email address: raul.juarez@unirioja.es

Background: The complications from coronavirus disease 2019 (COVID-19) have been the subject of study in diverse scientific reports. However, many aspects that influence the prognosis of the disease are still unknown, such as frailty, which inherently reduces resistance to disease and makes people more vulnerable. This study aimed to explore the complications of COVID-19 in patients admitted to a third-level hospital and to evaluate the relationship between these complications and frailty. Methods: An observational, descriptive, prospective study was performed in 2020. A sample of 254 patients from a database of 3112 patients admitted to a high-level hospital in Madrid, Spain was analyzed. To assess frailty (independent variable) the Clinical Frailty Scale (CFS) was used. The outcome variables were sociodemographic and clinical, which included complications, length of stay, intensive care unit admission, and prognosis. Results: $13.39 \%$ of the patients were pre-frail and $17.32 \%$ were frail. Frail individuals had a shorter hospital stay, less intensive care unit admission, higher mortality, and delirium, with statistical significance. Conclusion: Frailty assessment is a crucial approach in patients with COVID-19, given a higher mortality rate has been demonstrated amongst frail patients. The CFS could be a predictor of mortality in COVID-19. 


\title{
1 Outcomes of hospitalized patients with COVID-19 2 according to level of frailty
}

3

4

5

6

7

8

\author{
Eva María Andrés-Esteban ${ }^{1}$, Manuel Quintana-Diaz", Karen Lizzette Ramírez-Cervantes ${ }^{3,}$ Irene \\ Benayas Peña ${ }^{4}$, Alberto Silva-Obregón ${ }^{5}$, Rosa Magallón Botaya ${ }^{6}$, Ivan-Santolalla-Arnedo ${ }^{7}$, Raúl Juárez- \\ Vela $^{8^{*}}$ and Vicente Gea-Caballero ${ }^{9}$ \\ 1 Universidad Rey Juan Carlos, Madrid, Spain. Grupo PBM. Instituto de Investigación-IdiPaz, Madrid, Spain; \\ e.andres@live.com \\ 2 Servicio de Medicina Intensiva. Hospital Universitario La Paz (Madrid), Grupo PBM. Instituto de \\ Investigación-IdiPaz, Madrid, Spain; mquintanadiaz@gmail.com \\ 3 Departamento de Prevención. Asociación Española contra el Cáncer. Madrid, Spain; Grupo PBM. Instituto de \\ Investigación-IdiPaz, Madrid, Spain; karenlizzetteramirez@gmail.com \\ 4 Investigador Instituto de Investigación Hospital Universitario La Paz. Madrid, Spain; irene33_m@hotmail.com \\ 5 Servicio de Medicina Intensiva. Hospital de Guadalajara, Guadalajara, Spain. Grupo PBM. Instituto de \\ Investigación-IdiPaz, Madrid, Spain; jasoeres@gmail.com \\ 6 Instituto Aragonés de Ciencias de la Salud. Universidad de Zaragoza. Zaragoza, Spain; med000764@gmail.com \\ 7 Universidad de La Rioja-Centro de Investigación Biomédica de La Rioja CIBIR- Logroño. La Rioja, Spain; \\ raul.juarez@unirioja.es \\ 8 Universidad de La Rioja- Centro de Investigación Biomédica de La Rioja CIBIR- Logroño. La Rioja, Spain; \\ ivan.santolalla@unirioja.es \\ 9 Nursing School La Fe, adscript center of Universidad de Valencia, Spain. Research Group GREIACC, Health \\ Research Institute La Fe. Pabellón Docente, Torre H. Valencia, Spain; gea_vic@gva.es
}

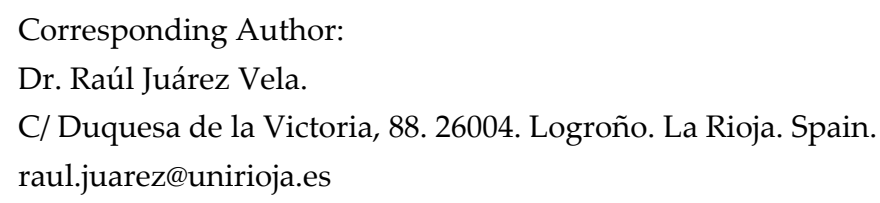

Abstract. Background: The complications from coronavirus disease 2019 (COVID-19) have been subject of study in diverse scientific reports. However, many aspects that influence the prognosis of the disease are still unknown, such as frailty, which inherently reduces resistance to disease and makes people more vulnerable. Objective: to explore the outcomes of patients with COVID-19 according to their degree of frailty and to study the association between frailty and death. Methods: An observational, descriptive, retrospective study was performed in 2020. A sample of 254 patients with COVID-19 was obtained from a database of 3112 patients >18-year-old admitted to a high-level hospital in Madrid, Spain. Frailty (independent variable) was assessed using the Clinical Frailty Scale (CFS), which classifies patients as robust (score <3), pre-frail (score 4) and frail (score $>5$ ). The analyzed outcomes were demographic and clinical features, including COVID-19 manifestations and complications, length of hospital stay, intensive care unit admission, and mortality rate. Results: $13.39 \%$ of the patients were pre-frail and $17.32 \%$ were frail. Frail individuals had a higher mortality rate (HR: 1.39, CI95\% 1.07-1.81), less intensive care unit admission $(\mathrm{p}=0.014)$, and a higher frequency of delirium $(\mathrm{p}=<0.001)$. Conclusion: Frailty assessment is a crucial approach in patients with COVID-19. The CFS could be an efficient tool to predict mortality in COVID-19. 
44 Keywords: Aged; Coronavirus infection; COVID-19; SARS-CoV-2; Mortality; Complications; Prognosis; 45 Frailty.

46

47

48

49

50

51

52

53

54

55

56

57

58

59

60

61

62

63

64

65

66

67

68

69

70

71

72

73

74

75

76

77

78

79

80

81

82

83

84

85

86

87

88

89

\section{Introduction}

The novel severe acute respiratory syndrome coronavirus 2 (SARS-CoV-2) and the consequential coronavirus disease 2019 (COVID-19) have been detected in more than 99.6 million people worldwide and have caused more than 2.1 million deaths. As of January 24, 2020, 2,6 million cases had been diagnosed in Spain and 55,441 people had died from the disease (Worldmeters, 2020).

In Spain most cases of COVID-19 have occurred in people aged 60 or above and fatality rates have showed to rise exponentially with age. In addition, the hospitalized people aged $>80$ account for $28.7 \%$ of admissions, reaching the highest mortality; in Italy (one of the countries with the highest infection and mortality rates in the world), until July, mortality in the population group $>80$ years was as high as almost $60 \%$. (Sornette et al, 2020). In the elderly, the presence of comorbid conditions has shown significant negative effects on prognosis (Maltese et al, 2020). In Europe, the first large cohort of hospitalized patients showed that older age and the presence of comorbidities were more common among subjects with fatal outcomes, both for the entire cohort and for those admitted to the Intensive Care Units (ICUs). However, the patients admitted to the ICU were older, had a higher male/female ratio and a higher prevalence of hypertension, obesity, diabetes mellitus and chronic obstructive pulmonary disease (Borobia et al, 2020).

Despite the presence of comorbidities increases the likelihood of death among patients with COVID19 (RR $(95 \%$ confidence interval $(\mathrm{CI})=1.69)$ (Abate et al, 2020); other characteristics such as frailty might predict poor prognosis (Hewitt et al., 2020). Frailty has been defined as "a medical syndrome with multiple causes and contributors that is characterized by diminished strength, endurance, and reduced physiologic function that increases an individual's vulnerability for developing increased dependency and/or death" (Morley, et al. 2013). Despite it has been related to disability, agedness and multimorbidity, they are not synonymous. For instance, frailty is more prevalent in older people, however, it can also affect the middleaged (Xue, 2011).

Several studies have described frailty as a poor outcome predictor in illness (Morley, et al. 2013), particularly in ICU patients (So et al, 2018; Muscedere et al, 2017; Bellelli et al, 2020). It has also been associated with more ICU admissions, higher in-hospital and ICU mortality rates, greater disability, and increased use of all forms of organ support (e.g., mechanical ventilation, noninvasive ventilation, vasopressors) (Zampieri et al, 2018). In the context of the current pandemic, a recent study has shown that frailty may account for as much as $49.4 \%$ of hospital COVID-19 admissions [Hewitt et al., 2020; Zampieri et al, 2018) and higher mortality rates and hospital stays have been described (Maltese et al, 2020). We have documented mortality that is clearly associated with age, rising drastically after 65 years of age, with an average of between $76-80$ years of age (approximately). (Sornette et al, 2020).

Stratifying patients according to their level of frailty is key to determine the risks to which they are exposed (Changfeng et al, 2019). Currently, a gold standard instrument for assessing frailty is lacking; however, the International Conference of Frailty and Sarcopenia Research Task Force recommends using a validated setting-specific instrument to screen frail elderly adults (Dent et al, 2019; Hoogendijk et al, 2019), such as the Clinical Frailty Scale (CFS).

In the United Kingdom (UK), the COVID-19 rapid action guide from the National Institute for Clinical Excellence (NICE) recommends the use of the CFS to assess frailty according to patient groups (Nice Guideline, 2020). This has generated controversy among some specialists, who argue that decisions should be made on an individual basis (Hewitt et al, 2020). However, frailty assessments employing the CFS has resulted to be an ai to decision-making regarding resources and patient services (Cesari et al, 2019). For instance, in primary care setting, frailty screening, together with the right assessment of illness severity

Peer] reviewing PDF | (2020:12:56252:2:0:NEW 14 Mar 2021) 
90

91

92

93

94

95

96

97

98

99

100

101

102

103

104

105

106

107

108

109

110

111

112

113

114

115

116

117

118

119

120

121

122

123

124

125

126

127

128

129

130

131

could be beneficial for the right allocation of patients avoiding excessive hospitalizations (Maltese et al, 2020). In addition, identifying frail and pre-frail individuals could improve the clinical management of this condition by focusing on the exhaustive monitoring and long recovery and rehabilitation that frail individuals may require (Hewitt et al, 2020). On the other hand, in the context of COVID-19 infection, the assessment of frailty could be a better determining factor for decision-making than age (Hewitt et al, 2020). Therefore, the rationale for assessing frailty in COVID-19 individuals is justified (Maltese et al, 2020).

Given that frailty could associate a high risk of complications, severe manifestations and death in COVID-19 patients, our study aimed at exploring the outcomes of patients admitted to a third-level hospital with COVID-19 according to the degree of frailty and to study the association between frailty and death. We hypothesize that, as previously reported, frail individuals will experience poorer health outcomes.

\section{Materials and Methods}

Design: We performed an observational, descriptive and retrospective

Population and sample: A total of 3581 clinical episodes were extracted from a database of 3112 patients from La Paz University Hospital (LPUH) during the period between 07/15/2020 and 07/31/2020, meaning a patient could have been admitted to the hospital several times. The database included sociodemographic data, clinical status, laboratory findings, and clinical management of the patients admitted with a respiratory infection by SARS-CoV-2 (determined by polymerase chain reaction, PCR) since the beginning of the current pandemic. A sample of 254 patients was randomly chosen from the total population, and with this sample size, in terms of the most frequent complications, a test power of at least 0.8744 was obtained.

Frailty assessment: Frailty was assessed by the CFS, which is a tool initially developed by the Canadian Health Study of Aging based on the Frailty Index (FI) to be used in patients older than 65 years. It was created with the objective of developing a simple instrument to stratify patients according to their degree of vulnerability and to relate it to patients' needs and prognoses. However, contrary to the FI, that includes comorbidity within its score, which has been independently associated with worse clinical outcomes in COVID-19 patients, the CFS does not take into account comorbidities for its assessment, allowing to focus on the investigation of the accurate relationship between frailty and clinical outcomes (Kow \& Hasan, 2020).

The CFS is a practical, efficient tool that assigns a score between 1 and 7 based on activity, functionality and disability (Rockwood et al. 2005). It classifies patients as robust (score 1-3), pre-frail (score 4), and frail (score $>5$ ) (Dent et al, 20019). This stratification allowed to identify not only frail individuals, who usually have higher risk of serious health complications and worse prognosis; but pre-frail subjects as well, who have reported an increased risk of negative events, such as an elevated mortality from all causes (Changfeng et al, 2019).

The CFS scale was not taken directly from the medical record but involved analyzing family medical history, nursing history, and in some cases interviewing the patient directly. Six doctors spent 15 days collecting this information and classifying patients according to the CFS scale. Parallelly, a group of experts trained in the use of frailty scales classified the same patients according to their level of frailty obtaining a Kappa 0,89 (IC 95\% 0.82-0.94)

Peer) reviewing PDF | (2020:12:56252:2:0:NEW 14 Mar 2021) 
132

133

134

135

136

137

138

139

140

141

142

143

144

145

146

147

148

149

150

151

152

153

154

155

156

157

158

159

160

161

162

163

164

165

166

167

168

169

170

171

172

\section{Variables}

Exposure variables

Frailty as measured by CFS. According to this scale, the levels of frailty range from robust (score 1), to those who are completely dependent on others (score 7), to terminal patients (score 9). A score of 5 or more is indicative of frailty, and a score of 4 is considered a pre-frailty state. Patients scoring 1-3 are considered healthy or robust (Rockwood et al, 2005).

Covariates (Secondary variables)

The data regarding secondary variables included demographic information (age, gender); previous medical history and comorbidities; clinical variables (from arrival at the emergency room to hospital admission); laboratory test findings; and diagnostic imaging performance and results.

\section{Outcome variables}

The main variables of the study were based on patients' outcomes (e.g., the presence of complications, need for ICU admission, days of stay, mortality) and on a frailty assessment using the CFS.

\section{Statistical analysis}

Quantitative variables were described using robust statistics, such as median-and interquartile interval , whereas for qualitative variables a frequency distribution was used. For the comparison of quantitative not normally distributed variables between frailty groups, the Kruskal-Wallis non-parametric $\mathrm{H}$ test was used, based on the Shapiro-Wilk test. Finally, for the comparison of qualitative variables, the chi-squared test was used.

The survival estimate was made using the Kaplan-Meier method comparing the survival curve between groups with the log-rank test. The multivariate analysis was carried out by means of Cox regression, with the forward conditional method, introducing as independent variables the CFS scale and the variables that obtained statistical significance in the bivariate analysis or that could have a clinically plausible implication. The results of the multivariate model were presented as a Hazard ratio $(95 \%$ confidence interval [CI]).

Statistical analysis was performed with STATA v16.0 and a pvalue of 5\% was considered statistically significant.

\section{Ethical considerations}

The study was performed in accordance with the principles of the Declaration of Helsinki (2008 update; available on the World Medical Association website-http://www.wma.net/e/policy/b3.htm) and in accordance with the standards of good clinical practice as described in the ICH Harmonized Tripartite Guidelines for Good Clinical Practice (1996), and the guidelines for Good Epidemiological Practice (http://www.ieaweb.org/GEP07.htm). The study was approved by the Clinical Research Ethics Committee of La Paz University Hospital, Madrid, with LPUH code: PI-4155. It was not necessary a formulary of informed consent due to the anonymized database was extracted.

This study was conducted in accordance with European and Spanish regulations for the protection of personal data (Organic Law 3/2008).

Peer] reviewing PDF | (2020:12:56252:2:0:NEW 14 Mar 2021) 
173

174

175

176

177

178

179

180

181

182

183

184

185

186

187

188

189

190

191

192

193

194

195

196

197

198

199

200

201

202

203

204

205

206

207

208

209

210

211

212

213

214

215

216

217

218

\section{Results}

Patients characteristics

Complete information for all variables was collected from 254 patients. According to the CFS instrument $69.29 \%$ were classified as robust (CFS=1-3), $13.39 \%$ as pre-frail (CFS= 4), and $17.32 \%$ as frail (CFS $>5$ ). Table 1 shows demographic and housing characteristics, COVID-19 contagion risks, level of dependency and previous comorbidities of the patients studied according to their level of frailty.

The individuals classified as frail were mostly women (56,82\%), with an older age (median[IQR]: 81.5 [7287.75]) compared with the robust (median[IQR]: 69.50 (55.00-79.00) and pre-fail (median[IQR]: 76.00 (66.50$82.50)$ subjects. Frail patients had a higher prevalence of suspected nosocomial transmission $(27.27 \%)$, compared with robust $(1.72 \%)$ or prefrail (3.03\%) individuals.

In table 1 it can also be observed that most comorbidities had a similar distribution among the 3 groups defined above, except for heart disease - for which the robust group had a very low prevalence compared with the pre-frail and frail individuals $(18.75 \%$ versus $41.18 \%$ and $40.91 \%$, respectively; $p=0.001)$ - and chronic bronchitis, for which the prevalence grew linearly among the 3 groups $(p=0.007)$. With the same pattern as heart disease, chronic renal disease was less prevalent among robust patients $(7.95 \%)$ compared to pre-frail and frail groups (20.95\% and $20.45 \%$, respectively; $p=0.017)$. Differences were also observed regarding the prevalence of previous neurological disorders, obesity, and dementia.

At arrival to the emergency room the clinical presentation of COVID-19 was similar among groups; however, as shown in Table 2, the highest incidence of altered behavior and level of consciousness occurred in frail patients $(11.93 \%, \mathrm{p}=<0.001)$

\section{COVID-19 outcomes}

Table 3 shows all the in-hospital complications related to COVID-19 according to level of frailty. The most common complications among patients with COVID-19 were acute renal failure (46 patients), delirium (38 patients), and acute respiratory distress syndrome (ARDS) (51 patients).

Despite the length of hospital stay was similar between groups $(\mathrm{p}=0.296)$, ICU admissions $(\mathrm{p}=0.014)$ were less prevalent among frail individuals. Moreover, mortality rates increased linearly among the 3 groups $(p=0.012)$.

A statistically significant difference was found regarding delirium, whose incidence was $8.52 \%$ in robust patients, $11.76 \%$ in pre-frail subjects and $43.18 \%$ in frail individuals. Delirium was also associated with a higher mortality rate, being presented in $27.30 \%$ of frail patients versus in $4.55 \%$ without it; $p<$ 0.001 .

No differences were found between groups regarding the rest of the COVID-19 outcomes shown in Table 3. However, it should be noticed that heart failure appeared as a complication of the disease in $5.88 \%$ and $6.82 \%$ of pre-frail and frail individuals, respectively, compared with an incidence of $1.14 \%$ in the robust group; nonetheless, this difference did not reach statistical significance $(p=0.059)$.

Figure 1 shows the Kaplan-Meier survival estimation curves for each group of patients, according to the CFS frailty scale. In this graph a huge difference during the first fortnight can be detected, especially for patients identified as frail. The differences observed were statistically significant (pvalue $<0.001$ ). Finally, a multivariate model was performed using a Cox regression model from the variables shown in the Table 4. The results demonstrated that frailty was associated with mortality (HR: 1.39, CI95\% 1.071.81). However, other factors such as chronic heart disease, chronic kidney disease, and high blood pressure were also associated with death.

Peer) reviewing PDF | (2020:12:56252:2:0:NEW 14 Mar 2021) 


\section{4. Discussion}

220 Our study introduces important findings. In this retrospective cohort study, we found that frailty was a

221

222

223

224

225

226

227

228

229

230

231

232

233

234

235

236

237

238

239

240

241

242

243

244

245

246

247

248

249

250

251

252

253

254

255

256

257

258

259 major predictor of mortality among hospitalized elderly patients with COVID-19; cardiovascular disease, chronic kidney disease, and hypertension were also shown to predict decreased survival in the fullyadjusted model of frailty.

By reporting the prevalence of the complications in a sample of 254 patients admitted with a diagnosis of COVID-19, we have contributed to increase the current knowledge regarding this new disease. The main findings of our study focused, on one hand, on the sociodemographic characteristics of individuals with COVID-19; and on the other hand, on the clinical symptoms at admission and in-hospital complications of these patients stratified by frailty profiles. Among the highlights of our clinical findings are the presentation of behavioral alterations and delirium, the higher mortality rate and the less ICU admission among frail individuals.

Sociodemographic characteristics of interest

In our study, we found that $17.32 \%$ of the patients were classified as frail, however, if the subjects with pre-frailty were considered, the total prevalence would increase to $30.71 \%$, which is still a much lower result compared with another study that have reported proportions of $39.6 \%-42.0 \%$ (Hewitt et al, 2020). Choi et al. demonstrated that the most affected frail patients were women and residents of nursing homes, which could be related to the advanced age and the dependency situation that feature these patients (Choi et al, 2015). In our study, old age, female sex, dependency, living in a nursing home, and having comorbidities were the high-risk profiles of frail individuals. These data are relevant because frailty is a condition that increases the risk of hospitalization, unwanted incidents, and mortality [Martínez-Reig et al, 2016).

The devastating effects of the pandemic on nursing homes are well known causing a disproportionate number of infections and deaths worldwide (Rutten et al, 2020). The typical response to prevent the transmission of the virus in nursing homes has been the aggressive isolation of patients, sometimes ignoring its possible collateral effects in patients with dementia, delirium, or other mental health problems, for whom human relationships are essential (Tan et al, 2020). It has been reported that in similar previous epidemics (SARS-CoV in 2003 and MERS-CoV in 2012) approximately 35\% of the survivors showed psychiatric disorders during their early recovery, including depression, stress, and anxiety, in addition to other minor symptoms (Mak et al, 2009).

The preexisting medical conditions that showed significant associations with frailty in our study were kidney disease, dementia, cardiovascular and respiratory diseases; therefore, the patients with COVID-19 could fit the typical profile of frail individuals: women, elderly, many of them institutionalized in nursing homes, and with comorbidities (Romero Cabrera, 2011). Comorbid conditions are also a notable element as a prognostic factor for the disease; some studies have especially highlighted advanced age, frailty, and the prior presence of chronic diseases (comorbidity) as important risk factors in COVID-19 (Landi et al, 2020). Hence, the control of comorbidities could be a key measure to improve COVID-19 outcomes, especially considering that a large proportion of deaths occurred during influenza pandemics were due to a decompensation of previous medical conditions and their subsequent complications, which is actually being reported during the current pandemic (Garnier-Crussard et al, 2020). 
261

262

263

264

265

266

267

268

269

270

271

272

273

274

275

276

277

278

279

280

281

282

283

284

285

286

287

288

289

290

291

292

293

294

295

296

297

298

299

300

301

302

303

304

305

306

307

Clinical symptoms and complications

Although CFS does not consider multimorbidity within its classification, it is known that they are related to worse outcomes. Our results showed a similar distribution of multimorbidity (without prioritizing any disease over another) among the three groups with some exceptions: neurological disease $(20.45 \%)$, dementia $(34.09 \%)$, heart disease $(40.91 \%)$, chronic bronchitis $(22.73 \%)$ and obesity $(13.64 \%)$ that were more prevalent among frail patients.

Some of the chronic pathologies that were associated with poor prognosis were chronic heart disease, chronic kidney disease and hypertension, which are frequent conditions among elderly and frail people that increase their vulnerability to suffering worse outcomes.

The relationship between frailty/multimorbidity, in view of the results, since multimorbidity and frailty may be bidirectionally related, should lead us to a deep reflection about discuss the role of a multidimensional approach integrating multimorbidity and frailty in the management of hospitalized patients (Boeckxstaens et al, 2015). As Price et al (2015) argue in their research protocol, it is important to know the association between multimorbidity and frailty with health outcomes, as this could enable better allocation of specialized resources, and especially holistic and shared decision making with patients.

It is well known that respiratory viruses, such as coronaviruses, can produce neuroinvasion and neurological symptoms, in addition to the severe hypoxia that respiratory symptoms can generate, especially in the elderly (Carod Artal, 2020). According to our results, neurological complications from COVID-19 were uncertain, with nonspecific and systemic symptoms (headaches, dizziness, etc.), highlighting the most common and more specific disorders of smell and taste. However, the most remarkable results observed in frail people were alterations of behavior and delirium (43.18\%). Mao et al. established that alterations in the level of consciousness can be severe in almost $15 \%$ of cases, with only $2.4 \%$ considered mild (Mao et al, 2020). However, our results were identical to Mcloughlin et al. (42\%) (Mcloughlin et al, 2020) and higher than that reported by Zazzara et al. (38\%) (Zazzara et al, 2020) Hence, an episode of delirium in elderly and frail patients could be the first symptom of COVID-19, as suggested by another author (O'Hanlon et al, 2020). In addition, since frailty implies physical and mental deterioration, these data highlight the importance of including sociopsychological support in our clinical management in order to reduce fatal outcomes among frail patients.

Frailty has been considered a predictor of mortality (Changfeng et al, 2019; Dent el al, 2019). In our study, in addition to confirming the relationship between frailty and mortality in patients with COVID-19 (HR: 1.39, C195\% 1.07-1.81), as previously hypothesized, delirium behaved as a predictor of death in frail people. Despite that another study (Mao et al, 2020) did not find an association between delirium and mortality, this complication is being studied and some studies have already offered clinical guidance for treating delirium in patients with COVID-19 in ICUs (Kotfis et al, 2020).

We also found, with statistical significance, a lower tendency for COVID-19 pneumonia to be mild in frail people. However, without statistical significance, a greater tendency for pneumonia to be severe in the groups of frail and pre-frail individuals was observed (in more than $60 \%$ of the patients of both groups pneumonia was severe, while in the robust group it occurred in $47.73 \%$ of the cases). These findings are concerning, given SARS-CoV-2 appears to be a potentially similar virus to SARS-CoV and MERS-CoV-6; in which, elderly patients had a greater tendency to develop serious respiratory diseases, greater complications, more disability, and higher mortality (Garnier-Crussard et al, 2020). However, these clinical overviews are similar to the findings of our study, which demonstrated an increased risk of developing severe pneumonia and a high risk of mortality for the elderly, frail, and those with multimorbidity (Weiss et al, 2020).

A previous study (Miles et al, 2020) has suggested that frailty might not be a good prognostic factor for death; however, our estimations showed a remarkable reduction in survival rates in frail patients which was not observed in prefrail and robust individuals. Other remarkable findings were the significant

Peer] reviewing PDF | (2020:12:56252:2:0:NEW 14 Mar 2021) 
308

309

310

311

312

313

314

315

316

317

318

319

320

321

322

323

324

325

326

327

328

329

330

331

332

333

334

335

336

337

338

339

340

341

342

343

344

345

346

347

348

\section{9}

350

351

difference observed regarding the need for ICU admission among frail subjects, and its association with mortality. It was concerning to find that the subgroup of frail people had a mortality rate close to $60 \%$, but only $11.36 \%$ were admitted to the ICU. Lower rates were obtained by Hewitt et al (Hewitt et al, 2020) who reported a mortality rate close to $50 \%$ in frail people from the UK, with $7 \%$ of terminal patients. However, it must be taken into consideration that during the initial period of the pandemic the burden of hospital strains triggered protocols of limited ICU admissions that discouraged patients who were not considered for resuscitation, i.e., patients with a poor prognosis; therefore, frail individuals could have also been discouraged from ICUs. In addition, due to frail subjects were older than pre-frail and robust individuals, it suggests that age could have been a determining factor in the decision-making of COVID-19 treatment and health resource allocation. Nevertheless, a recent study has concluded that even severely frail elderly patients with a poor prognosis can be benefited from hospitalization for COVID-19, and their survival could increase if enough resources were available for them (De Smet et al, 2020); therefore, the risk of worse outcomes and less ICU admission among the elderly could be better explored to avoid ageism in a setting of limited health resources.

This serious situation is not exclusive to Spain. When COVID-19 hospital admissions exceeded the availability of beds, hospitals in China and Italy (among other countries in Europe) also had to face the difficult task of classifying patients in order to allocate resources (Boreskie et al, 2020). Age was an important criterion in the triage of limited resources, as we observed (Boreskie et al, 2020); for example, in Italy, generating some rejection by equating old age with frailty (Rosenbaum, 2020). It is remarkable that this situation has affected countries with a very advanced life expectancy, such as Italy or Spain. In the UK, the criterion recommended by the NICE COVID-19 Quick Guide was the assessment of income frailty as a criterion to assist in decision-making in the allocation of resources, with less attention to biological age (NICE Guideline, 2020).

Assessing the level of frailty as a regulatory condition for the allocation of health resources is not new. Some economic studies have previously addressed this problem in response to the high demand for care of frail, elderly, and multimorbidity patients (Cesari et al, 2016). Therefore, it should not be surprising that when health services are strained - such as during the peaks in the COVID-19 pandemic- some patients are ruled out for ICU admission, as seen in our study and in other countries.

Expanding our knowledge about frailty in COVID-19 is essential. The relationship between frailty and mortality, ICU admission, and hospital stay appears clear. However, as a recently published in a systematic review, research should now be directed toward interventions and treatments, given the guidelines for care of frail patients with COVID-19 are not based on strong evidence. As previously addressed, the implications of COVID-19 for the physical and mental health of patients and health professionals should lead us to reflect on the need for developing specific care protocols, based on solid evidence, to facilitate optimal decision-making in vulnerable and frail individuals, especially in terms of the detection of early symptoms for the activation of healthcare processes (Ramírez-Ortiz et al, 2020)

\section{Limitations}

Given that our work was a descriptive observational study carried out in a random sample selected from a larger cohort of COVID-19 indivduals, we cannot demonstrate causality in the relationship between the variables studied. In addition, due to the CFS was determined by the revision of medical records there could be slight variability in the assessment when it is performed in a medical setting.

\section{Conclusions}

Frailty has shown to worsen the prognosis of COVID-19 patients. Higher frailty scores were associated with an increased mortality rate in hospitalized patients with COVID-19. The presence of delirium in

Peer] reviewing PDF | (2020:12:56252:2:0:NEW 14 Mar 2021) 
352

353

354

355

356

357

358

359

360

361

362

363

364

365

366

367

368

369

370

371

372

373

374

375

376

377

378

379

380

381

382

383

384

385

386

387

388

389

390

391

392

393

394

395

396

397

elderly and/or frail patients could be a frequent atypical manifestation of COVID-19 that could also predict a higher mortality rate. The assessment of frailty in hospital settings could be an efficient tool to avoid ageism, improve clinical decision-making and health resource allocation, especially due to frail individuals might require closer monitoring and/or more intensive treatment for their preexisting conditions. Due to frailty may affect both physical, physiological, and cognitive functions, frailty itself demands implementation of a holistic approach to patient care.

\section{Funding: ISCIII (COV20/00519) co-funded by ERDF " A way to make Europe"}

7. Acknowledgments: COVID@HULP Working Group of Hospital La Paz. To Silvia Erce, Raquel Carmona and Aitana López, for their help and support in this investigation.

8. Conflicts of Interest: The authors declare no conflicts of interest.

\section{References.}

Abate SM, Ahmed Ali S, Mantfardo, B, Basu B. 2020. Rate of Intensive Care Unit admission and outcomes among patients with coronavirus: A systematic review and Meta-analysis. PLoS One 15:e235653. DOI 10.1371/journal.pone.0235653.

Bellelli G, Rebora P, Valsecchi MG, Bonfanti P, Citerio G, COVID-19 Monza Team members. 2020. Frailty index predicts poor outcome in COVID-19 patients. Intensive care medicine 46(8):1634-1636. Available from: https://doi.org/10.1007/s00134-020-06087-2

Boeckxstaens P, Vaes B, Legrand D, Dalleur O, De Sutter A, \& Degryse JM. 2015. The relationship of multimorbidity with disability and frailty in the oldest patients: a cross-sectional analysis of three measures of multimorbidity in the BELFRAIL cohort. The European journal of general practice, 21(1), 39-44. https://doi.org/10.3109/13814788.2014.914167

Boreskie KF, Boreskie PE, Melady D. 2020. Age is just a number - and so is frailty: Strategies to inform resource allocation during the COVID-19 pandemic. Canadian Journal of Emergency Medicine, 22: 4. DOI: 10.1017/cem.2020.358

Borobia AM, Carcas AJ, Arnalich F, Álvarez-Sala R, Monserrat-Villatoro J, Quintana M, Figueira JC, Torres SantosOlmo RM, García-Rodríguez J, Martín-Vega A, Buño A, Ramírez E, Martínez-Alés G, García-Arenzana N, Núñez MC, Martí-de-Gracia M, Moreno Ramos F, Reinoso-Barbero F, Martin-Quiros A, Rivera Núñez A, Mingorance J, Carpio Segura CJ, Prieto Arribas D, Rey Cuevas E, Prados Sánchez C, Rios JJ, Hernán MA, Frías J, Arribas JR. On Behalf Of The Covid Hulp, Working Group. 2020. A Cohort of Patients with COVID-19 in a Major Teaching Hospital in Europe. J Clin Med 9. DOI 10.3390/jcm9061733.

Carod Artal FJ. 2020. Complicaciones neurológicas por coronavirus y COVID-19. Revista de neurologia 70:311. DOI 10.33588/rn.7009.2020179.

Cesari M, Costa N, Hoogendijk EO, Vellas B, Canevelli M, Pérez-Zepeda MU. 2016. How the frailty index may support the allocation of health care resources: an example from the INCUR study. J Am Med Dir Assoc 17:448-450. Available from: https://www.jamda.com/article/S1525-8610(16)00093-1/fulltext

Changfeng M, Shouyan X, Yu F. 2019. Frailty for predicting all-cause mortality in elderly acute coronary syndrome patients: A meta-analysis. Ageing Research Reviews 52:1-6. DOI https://doi.org/10.1016/j.arr.2019.03.003. Available online: http://www.sciencedirect.com/science/article/pii/S1568163719300315)

Choi J, Ahn A, Kim S. 2015. Won Global prevalence of physical frailty by Fried's criteria in community-dwelling elderly with national population-based surveys. J Am Med Dir 16:548- 550. DOI 10.1016/j.jamda.2015.02.004.

Chong E, Chan M, Tan HN, Lim WS. 2020. COVID-19: Use of the Clinical Frailty Scale for Critical Care Decisions. Journal of the American Geriatrics Society 68(6):E30-E32. DOI: https://doi.org/10.1111/igs.16528

De Smet R, Mellaerts B, Vandewinckele H, Lybeert P, Frans E, Ombelet S, Lemahieu W, Symons R, Ho E, Frans J, Smismans A, Laurent MR. 2020. Frailty and Mortality in Hospitalized Older Adults With COVID-19: Retrospective

Peer] reviewing PDF | (2020:12:56252:2:0:NEW 14 Mar 2021) 
398

399

400

401

402

403

404

405

406

407

408

409

410

411

412

413

414

415

416

417

418

419

420

421

422

423

424

425

426

427

428

429

430

431

432

433

434

435

436

437

438

439

440

441

442

443

444

445

446

Observational Study. Journal of the American Medical Directors Association 21:928-932.e1. DOI 10.1016/j.jamda.2020.06.008. Available online: http://dx.doi.org/10.1016/j.jamda.2020.06.008.

Dent E, Morley JE, Cruz-Jentoft A, Woodhouse L, Rodríguez-Mañas L, Frie, LP, ... Vellas, B. 2019. Physical Frailty: ICFSR International Clinical Practice Guidelines for Identification and Management. J Nutr Health Aging 23:771-787, DOI 10.1007/s12603-019-1273-z.

Garnier-Crussard A, Forestier E, Gilbert T, Krolak-Salmon P. 2020. Novel Coronavirus (COVID-19) Epidemic: What Are the Risks for Older Patients? Journal of the American Geriatrics Society 68:939-940. DOI 10.1111/jgs.16407.

Hewitt J, Carter B, Vilches-Moraga A, Quinn TJ, Braude P, Verduri A... McCarthy, K.; Collaborators, C.S. 2020. The effect of frailty on survival in patients with COVID-19 (COPE): a multicentre, European, observational cohort study. Lancet Public Health 5:e444-e451. DOI 10.1016/S2468-2667(20)30146-8.

Hoogendijk EO, Afilalo J, Ensrud KE, Kowal P, Onder G, Fried LP. 2019. Frailty: implications for clinical practice and public health. Lancet 394:1365-1375. DOI 10.1016/S0140-6736(19)31786-6.

Kotfis K, Williams Roberson S, Wilson JE, Dabrowski W, Pun BT, Ely EW. 2020. COVID-19: ICU delirium management during SARS-CoV-2 pandemic. Critical care 24:1-17. DOI 10.1186/s13054-020-02882-x.

Kow CS, Hasan SS. 2020. Role of frailty in COVID-19 patients. Intensive care medicine 1-2. Advance online publication. DOI: https://doi.org/10.1007/s00134-020-06172-6

Landi F, Barillaro C, Bellieni A, Brandi V, Carfi A... Bernabei, R. 2020. The New Challenge of Geriatrics: Saving Frail Older People from the SARS-COV-2 Pandemic Infection. The Journal of nutrition, health \& aging 24:466-470. DOI 10.1007/s12603-020-1356-x.

Mak IWC, Chu CM, Pan PC, Yiu MGC, Chan VL. 2009. Long-term psychiatric morbidities among SARS survivors. General Hospital Psychiatry 31:318-326. DOI 10.1016/j.genhosppsych.2009.03.001.

Maltese G, Corsonello A, Di Rosa M, Soraci L, Vitale C, Corica F, Lattanzio F. 2020. Frailty and COVID-19: A Systematic Scoping Review. Journal of clinical medicine 9(7):E2106. DOI: https://doi.org/10.3390/jcm9072106

Mao L, Jin H, Wang M, Hu Y, Chen S... Hu B. 2020. Neurologic Manifestations of Hospitalized Patients With Coronavirus Disease 2019 in Wuhan, China. JAMA neurology 77:683-690. DOI 10.1001/jamaneurol.2020.1127.

Martínez-Reig M, Flores Ruano T, Fernández Sánchez M Noguerón García A, Romero Rizos L, Abizanda Soler P. 2016. Frailty and long-term mortality, disability and hospitalisation in Spanish older adults. The FRADEA Study. Rev Esp Geriatr Gerontol 51:254-259, DOI: 10.1016/j.regg.2016.01.006.

Mcloughlin BC, Miles A, Webb TE, Knopp P, Eyres C... Davis D. 2020. Functional and cognitive outcomes after COVID19 delirium. European geriatric medicine 1-6. DOI: 10.1007/s41999-020-00353-8.

Miles A, Webb TE, Mcloughlin BC, Mannan I, Rather A, Knopp P, Davis D. 2020. Outcomes from COVID-19 across the range of frailty: excess mortality in fitter older people. European geriatric medicine 1-5. DOI: 10.1007/s41999-020-00354-7. Available online: https://search.proquest.com/docview/2425590119.

Morley JE, Vellas B, van Kan GA, Anker SD, Bauer JM... Walston J. 2013. Frailty consensus: a call to action. Journal of the American Medical Directors Association 14(6):392-397. DOI: https://doi.org/10.1016/j.jamda.2013.03.022

Muscedere J, Waters B, Varambally A, Bagshaw SM, Boyd JG... Rockwood K. 2017. The impact of frailty on intensive care unit outcomes: a systematic review and meta-analysis. Intensive care medicine 43(8):1105-1122. DOI: https://doi.org/10.1007/s00134-017-4867-0

NICE Guideline, 2020. COVID-19 rapid guideline: critical care I Guidance I NICE [Internet]. National Institute for Clinical Excellence Guidelines. [cited 2020 Sept 19]. Available from: https://www.nice.org.uk/guidance/ng159

O'Hanlon S, Inouye SK. 2020. Delirium: a missing piece in the COVID-19 pandemic puzzle. Age and ageing 49:497-498, DOI 10.1093/ageing/afaa094.

Price, A., Barlow-Pay, F., Duffy, S., Pearce, L., Vilches-Moraga, A., Moug, S., Quinn, T., Stechman, M., Braude, P., Mitchell, E., Myint, P. K., Verduri, A., McCarthy, K., Carter, B., Hewitt, J., \& COPE Study Collaborators (2020). Study protocol for the COPE study: COVID-19 in Older PEople: the influence of frailty and multimorbidity on survival. A multicentre, European observational study. BMJ open, 10(9), e040569. https://doi.org/10.1136/bmjopen-2020-040569

Ramírez-Ortiz J, Castro-Quintero D, Lerma-Córdoba C, Yela-Ceballos F, Escobar-Córdoba F. 2020. Consequences of the COVID-19 pandemic in mental health associated with social isolation-. Health Sciences (preprint). Available online: https://preprints.scielo.org/index.php/scielo/preprint/view/303. 
447 Rockwood K, Song X, MacKnight C, Bergman H, Hogan DB, McDowell I, Mitnitski A. 2005. A global clinical measure 448 of fitness and frailty in elderly people. CMAJ: Canadian Medical Association journal 173(5):489-495. DOI: 449 https://doi.org/10.1503/cmaj.050051

450 Romero Cabrera AJ. 2011. Fragilidad y enfermedades crónicas en los adultos mayores. Medicina interna de México 27:456. 451 Available online: https://www.medigraphic.com/cgi-bin/new/resumen.cgi?IDARTICULO=31195.

452 Rosenbaum L. (2020). Facing Covid-19 in Italy - Ethics, Logistics, and Therapeutics on the Epidemic's Front Line. N 453 Engl J Med 382(20):1873-1875. DOI: https://doi.org/10.1056/NEJMp2005492

454 Rutten JJS, van Loon AM, Joling KJ, Smalbrugge M, van Buul LW, Hertogh CM. 2020. Ned Tijdschr Geneeskd 164. 455 Available online: https://www.ncbi.nlm.nih.gov/pubmed/32779925.

456 So R, Bannard-Smith J, Subbe CP, Jones DA, van Rosmalen J, Lighthall GK, \& METHOD study investigators. 2018. The 457 association of clinical frailty with outcomes of patients reviewed by rapid response teams: an international prospective observational cohort study. Critical care 22(1):227. DOI: https://doi.org/10.1186/s13054-018-2136-4 Sornette D, Mearns E, Schatz M. Ke W, Didier D. 2020. Interpreting, analysing and modelling COVID-19 mortality data. Nonlinear Dyn 101:1751-1776. DOI: https://doi.org/10.1007/s11071-020-05966-z

Tan LF, Seetharaman S. 2020. Preventing the Spread of COVID-19 to Nursing Homes: Experience from a Singapore Geriatric Centre. J Am Geriatr Soc 68:942. DOI: 10.1111/jgs.16447.

Weiss P, Murdoch DR. Clinical course and mortality risk of severe COVID-19. The Lancet 395:1014-1015. DOI: 10.1016/s0140-6736(20)30633-4.

465 Worldmeters, 2020. Coronavirus outbreak. [Accesed November 2, 2020]. Available online: 466 https://www.worldometers.info/coronavirus/.

467 Xue QL. 2011. The frailty syndrome: definition and natural history. Clin Geriatr Med 27:1-15. DOI: 468 10.1016/j.cger.2010.08.009.

469 Zampieri FG, Iwashyna TJ, Viglianti EM eTaniguchi LU, Viana WN, Costa R, Corrêa TD, Moreira CEN, Maia MO, 470 Moralez GM, Lisboa T, Ferez MA, Freitas CEF, de Carvalho CB, Mazza BF, Lima MFA, Ramos GV, Silva AR, Bozza 471 FA, Salluh JIF, Soares M; Orchestra Study 2018. Association of frailty with short-term outcomes, organ support and 472 resource use in critically ill patients. Intensive Care Med 44:1512-1520. DOI: https://doi.org/10.1007/s00134-018-5342-2.

473 Zazzara MB, Penfold R, Roberts A, Lee K, Dooley H... Ni Lochlainn M. 2020. Delirium is a presenting symptom of 474 COVID-19 in frail, older adults: a cohort study of 322 hospitalised and 535 community-based older adults. Med Rxiv 475 (preprint). DOI: https://doi.org/10.1101/2020.06.15.20131722 
Figure 1

Figure 1

Overall Survival

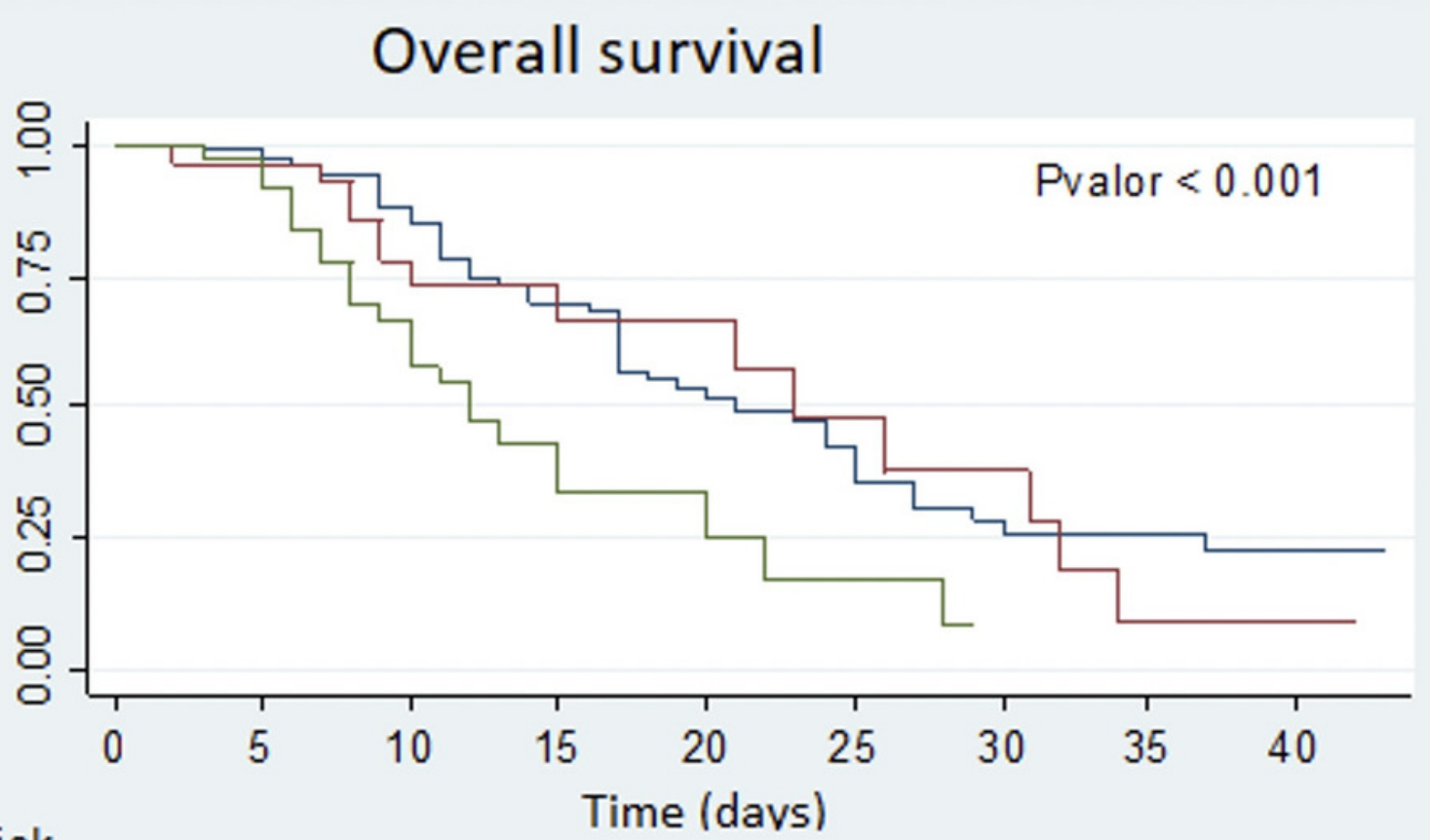

Number at risk

Robust

Pre-frail

$\begin{array}{lll}146 & 142 & 91\end{array}$

$\begin{array}{lll}29 & 28 & 18\end{array}$

Frail

$38 \quad 35$

50

10

22

9

$\begin{array}{ll}27 & 19\end{array}$

7

5

4

2

11

4

Robust Frail 


\section{Table $\mathbf{1}$ (on next page)}

Demographic Characteristics

Demographic and housing characteristics, COVID-19 contagion risks, level of dependency and previous comorbidities according to level of frailty 
Table 1. Demographic and housing characteristics, COVID-19 contagion risks, level of dependency and previous comorbidities according to level of frailty

\begin{tabular}{|c|c|c|c|c|}
\hline \multirow{2}{*}{ Variable } & \multicolumn{3}{|c|}{ CFS } & \multirow{2}{*}{ p-valu } \\
\hline & Robust (CFS $\leq 3$ ) & Pre-Frail $(C F S=4)$ & Frail (CFS $\geq 5$ ) & \\
\hline & $176(69.29 \%)$ & $34(13.39 \%)$ & $44(17.32 \%)$ & \\
\hline Sex & & & & 0.018 \\
\hline Men & $113(69.94 \%)$ & $23(69.70 \%)$ & $19(43.18 \%)$ & \\
\hline Women & $61(35.06 \%)$ & $10(30.30 \%)$ & $25(56.82 \%)$ & \\
\hline Age (median, IQI) & $69.50(55.00-79.00)$ & $76.00(66.50-82.50)$ & $\begin{array}{l}81.50(72.00- \\
87.75)\end{array}$ & $<0.001$ \\
\hline Smoker & $13(7.39 \%)$ & $3(8.82 \%)$ & $3(6.82 \%)$ & 0.942 \\
\hline Health Care Provider & $9(5.77 \%)$ & $1(3.23 \%)$ & $0(0.00 \%)$ & 0.260 \\
\hline \multicolumn{5}{|l|}{ Housing conditions } \\
\hline & & & & $<0.001$ \\
\hline Not Overcrowding & $170(97.70 \%)$ & $32(96.97 \%)$ & $32(72.73 \%)$ & \\
\hline Nursing home & $3(1.72 \%)$ & $1(3.03 \%)$ & $12(27.27 \%)$ & \\
\hline Shelter & $1(0.57 \%)$ & $0(0.00 \%)$ & $0(0.00 \%)$ & \\
\hline \multicolumn{5}{|l|}{ COVID-19 contagion risks } \\
\hline $\begin{array}{l}\text { Direct/close contact with an individual } \\
\text { with COVID-19 }\end{array}$ & $21(13.91 \%)$ & $3(10.34 \%)$ & $7(17.95 \%)$ & 0.665 \\
\hline Nosocomial infection* & $19(11.11 \%)$ & $7(21.88 \%)$ & $14(31.82 \%)$ & 0.003 \\
\hline \multicolumn{5}{|l|}{ Level of dependency } \\
\hline & & & & $<0.001$ \\
\hline Dependent & $3(1.89 \%)$ & $1(3.23 \%)$ & $13(30.95 \%)$ & \\
\hline Semi-dependent & $1(0.63 \%)$ & $3(9.68 \%)$ & $5(11.90 \%)$ & \\
\hline Independent & $155(97.48 \%)$ & $27(87.10 \%)$ & $24(57.14 \%)$ & \\
\hline \multicolumn{5}{|l|}{ Comorbidities } \\
\hline Heart disease & $33(18.75 \%)$ & $14(41.18 \%)$ & $18(40.91 \%)$ & 0.001 \\
\hline High blood pressure & $85(48.30 \%)$ & $19(55.88 \%)$ & $30(68.18 \%)$ & 0.057 \\
\hline Chronic obstructive pulmonary disease & $14(7.95 \%)$ & $6(17.65 \%)$ & $5(11.36 \%)$ & 0.206 \\
\hline $\begin{array}{l}\text { (COPD) } \\
\text {-Chronic bronchitis }\end{array}$ & $12(6.82 \%)$ & $4(11.76 \%)$ & $10(22.73 \%)$ & 0.007 \\
\hline Asthma & $9(5.11 \%)$ & $4(11.76 \%)$ & $2(4.55 \%)$ & 0.294 \\
\hline Chronic kidney disease & $14(7.95 \%)$ & $7(20.95 \%)$ & $9(20.45 \%)$ & 0.017 \\
\hline Moderate kidney damage & $1(0.57 \%)$ & $2(5.88 \%)$ & $6(13.64 \%)$ & $<0.001$ \\
\hline Mild liver disease & $7(3.98 \%)$ & $0(0.00 \%)$ & $0(0.00 \%)$ & 0.203 \\
\hline Chronic neurological disorder & $9(5.11 \%)$ & $7(20.95 \%)$ & $9(20.45 \%)$ & 0.001 \\
\hline Malignant neoplasm & $21(11.93 \%)$ & $6(17.65 \%)$ & $9(20.45 \%)$ & 0.288 \\
\hline Chronic hematological disease & $11(6.25 \%)$ & $6(17.65 \%)$ & $4(9.09 \%)$ & 0.085 \\
\hline Obesity & $20(11.36 \%)$ & $10(29.41 \%)$ & $6(13.64 \%)$ & 0.022 \\
\hline Diabetes & $40(22.73 \%)$ & $10(29.41 \%)$ & $16(36.36 \%)$ & 0.162 \\
\hline Rheumatological disorder & $21(11.93 \%)$ & $4(11.76 \%)$ & $8(18.18 \%)$ & 0.530 \\
\hline Dementia & $1(0.57 \%)$ & $1(2.94 \%)$ & $15(34.09 \%)$ & $<0.001$ \\
\hline Dyslipidemia & $73(41.48 \%)$ & $18(52.94 \%)$ & $25(56.82 \%)$ & 0.124 \\
\hline $\begin{array}{c}\text { Common mental disorder (anxiety, } \\
\text { depression) }\end{array}$ & $17(9.66 \%)$ & $4(11.76 \%)$ & $10(22.73 \%)$ & 0.060 \\
\hline $\begin{array}{l}\text { Severe mental disorder (schizophrenia, } \\
\text { bipolar disorder, other) }\end{array}$ & $1(0.57 \%)$ & $0(0.00 \%)$ & $1(2.27 \%)$ & 0.445 \\
\hline
\end{tabular}

Note: the individuals with nosocomial infection risks included healthcare workers; patients who were hospitalized for another reason; and subjects who visited a relative/patient at a hospital before the pandemic declaration. 
Table 2 (on next page)

\section{Clinical symptoms}

We aprreciate the clinical symtomps at arrival to the emergency department according to level of frailty. 
Table 2. Clinical symptoms at arrival to the emergency department according to level of frailty.

\begin{tabular}{|c|c|c|c|c|}
\hline \multirow{2}{*}{ Symptoms and evolution } & \multicolumn{3}{|c|}{ Clinical frailty scale (CFS) } & \multirow[t]{2}{*}{ p-value } \\
\hline & Robust (CFS $\leq 3$ ) & Pre-frail $(C F S=4)$ & Frail (CFS $\geq 5$ ) & \\
\hline Fever & $140(79.55 \%)$ & $31(91.18 \%)$ & $31(72.73 \%)$ & 0.128 \\
\hline Headache & $21(11.93 \%)$ & $5(14.71 \%)$ & $4(9.09 \%)$ & 0.745 \\
\hline General discomfort & $76(43.18 \%)$ & $8(23.53 \%)$ & $21(47.73 \%)$ & 0.066 \\
\hline Myalgia & $38(21.59 \%)$ & $7(20.59 \%)$ & $3(6.82 \%)$ & 0.079 \\
\hline Rhinorrhea & $9(5.11 \%)$ & $3(8.82 \%)$ & $0(0.00 \%)$ & 0.173 \\
\hline Dysgeusia & $2(1.14 \%)$ & $0(0.00 \%)$ & $1(2.27 \%)$ & 0.651 \\
\hline Anosmia & $1(0.57 \%)$ & $0(0.00 \%)$ & $0(0.00 \%)$ & 0.801 \\
\hline Cough & $110(62.50 \%)$ & $20(58.82 \%)$ & $20(45.45 \%)$ & 0.121 \\
\hline Productive cough & $40(22.73 \%)$ & $12(35.29 \%)$ & $10(22.73 \%)$ & 0.284 \\
\hline Odynophagia & $12(6.82 \%)$ & $1(2.94 \%)$ & $0(0.00 \%)$ & 0.153 \\
\hline Chest pain & $15(8.52 \%)$ & $4(11.76 \%)$ & $3(6.82 \%)$ & 0.738 \\
\hline Costal pain & $6(3.41 \%)$ & $1(2.94 \%)$ & $0(0.00 \%)$ & 0.465 \\
\hline Hemoptoic expectoration & $1(0.57 \%)$ & $0(0.00 \%)$ & $2(4.55 \%)$ & 0.073 \\
\hline Dyspnea & $89(50.57 \%)$ & $16(47.06 \%)$ & $25(56.82 \%)$ & 0.665 \\
\hline Abdominal pain & $16(9.09 \%)$ & $2(5.88 \%)$ & $5(11.36 \%)$ & 0.704 \\
\hline Diarrhea & $36(20.45 \%)$ & $5(14.71 \%)$ & $6(13.64 \%)$ & 0.482 \\
\hline Nausea & $22(12.50 \%)$ & $2(5.88 \%)$ & $4(9.09 \%)$ & 0.478 \\
\hline Vomiting & $7(3.98 \%)$ & $2(5.88 \%)$ & $2(4.55 \%)$ & 0.880 \\
\hline Altered level of consciousness* & $21(11.93 \%)$ & $2(5.88 \%)$ & $14(31.82 \%)$ & $<0.001$ \\
\hline Altered behavior & $6(3.41 \%)$ & $0(0.00 \%)$ & $7(15.91 \%)$ & $<0.001$ \\
\hline
\end{tabular}

Note: These data were gathered from medical records. ${ }^{*}$ The assessment of level of consciousness and behavior was performed by a medical team at the emergency room. If necessary, further information was obtained from the person accompanying the patient at arrival. 


\section{Table 3(on next page)}

\section{Complications}

Complications observed during hospital admission according to level of frailty. 
Table 3. Complications observed during hospital admission according to level of frailty.

\begin{tabular}{ccccc} 
Complications & \multicolumn{2}{c}{ Clinical frailty scale (CFS) } & p-value \\
& $\begin{array}{c}\text { Robust } \\
\text { (CFS }\end{array}$ & Pre-Frail & Frail & \\
(CFS 4$)$ & (CFS $\geq 5)$ & \\
Noncomplicated disease & $32(18.18 \%)$ & $4(11.76 \%)$ & $8(18.18 \%)$ & 0.655 \\
Mild pneumonia & $65(39.93 \%)$ & $8(23.54 \%)$ & $8(18.18 \%)$ & 0.031 \\
Severe pneumonia & $84(47.73 \%)$ & $21(61.76 \%)$ & $29(65.91 \%)$ & 0.051 \\
Myocarditis & $35(19.89 \%)$ & $8(23.53 \%)$ & $8(18.18 \%)$ & 0.837 \\
Pericarditis & $1(0.57 \%)$ & $0(0.00 \%)$ & $0(0.00 \%)$ & 0.801 \\
Arrhythmia & $1(0.57 \%)$ & $0(0.00 \%)$ & $0(0.00 \%)$ & 0.801 \\
Cardiac ischemia & $6(3.41 \%)$ & $2(5.88 \%)$ & $2(4.55 \%)$ & 0.774 \\
Cardiac arrest & $2(1.14 \%)$ & $0(0.00 \%)$ & $0(0.00 \%)$ & 0.640 \\
Bacteremia & $12(6.82 \%)$ & $0(0.00 \%)$ & $4(9.09 \%)$ & 0.229 \\
Coagulation disorder & $14(7.95 \%)$ & $2(5.88 \%)$ & $4(9.09 \%)$ & 0.871 \\
Subsidiary anemia & $17(9.66 \%)$ & $5(14.71 \%)$ & $3(6.82 \%)$ & 0.505 \\
Rhabdomyolysis & $7(3.98 \%)$ & $1(2.94 \%)$ & $2(4.55 \%)$ & 0.936 \\
Acute kidney failure & $4(2.27 \%)$ & $0(0.00 \%)$ & $1(2.27 \%)$ & 0.674 \\
Pancreatitis & $28(15.91 \%)$ & $6(17.65 \%)$ & $12(27.27 \%)$ & 0.215 \\
Liver failure & $0(0.00 \%)$ & $0(0.00 \%)$ & $1(2.27 \%)$ & 0.091 \\
Delirium & $6(3.41 \%)$ & $0(0.00 \%)$ & $2(4.55 \%)$ & 0.490 \\
Psychiatric complications & $15(8.52 \%)$ & $4(11.76 \%)$ & $19(43.18 \%)$ & $<0.001$ \\
Adverse drug reaction & $6(3.41 \%)$ & $1(2.94 \%)$ & $1(2.27 \%)$ & 0.926 \\
Serious adverse drug reaction & $15(8.52 \%)$ & $5(14.71 \%)$ & $1(2.27 \%)$ & 0.138 \\
Sepsis & $2(1.14 \%)$ & $1(2.94 \%)$ & $1(2.27 \%)$ & 0.682 \\
Septic shock & $7(3.98 \%)$ & $3(8.82 \%)$ & $3(6.82 \%)$ & 0.429 \\
Intensive care unit admission & $12(6.82 \%)$ & $2(5.88 \%)$ & $3(6.82 \%)$ & 0.980 \\
Death & $55(31.25 \%)$ & $13(38.24 \%)$ & $5(11.36 \%)$ & 0.014 \\
Length of hospital stay (LOS) (median; IQR) & $62(35.23 \%)$ & $16(47.06 \%)$ & $26(59.09 \%)$ & 0.012 \\
LOS of survivors (median; IQR) & $11(9-19)$ & $10(7-14)$ & $5(1-11)$ & 0.296 \\
(2) & $12(17-23)$ & $14(18-27)$ & $10(13-28)$ & 0.006
\end{tabular}




\section{Table 4(on next page)}

Table 4

Cox regression according to CFS. 
Table 4. Cox regression according to CFS.*

\begin{tabular}{ccccc}
\hline & & & \multicolumn{2}{c}{ CI (95\%) } \\
\cline { 5 - 6 } & HR & p value & lower & upper \\
\hline Frail vs pre-frail/robust & 1.39 & 0.014 & 1.07 & 1.81 \\
Chronic heart disease & 1.68 & 0.035 & 1.04 & 2.72 \\
Chronic kidney disease & 2.08 & 0.018 & 1.13 & 3.81 \\
Hypertension & 1.51 & 0.049 & 1.00 & 2.38 \\
\hline C - Harrell = 0.681 & & & & \\
\hline
\end{tabular}

*Sex, Age, Overcrowding, Nosocomial Infection, Dependency, Heart Disease, COPD , Asthma, Kidney Disease, Neurological Disorder, Obesity, Dementia, ICU Admission, Hypertension, Mortality, and Frailty were the variables included in the multivariable model. 